\title{
Rasche Krankheitsprogession, frühe Insulinpflicht
}

\section{Wenn Kinder und Jugendliche einen Diabetes entwickeln, kann man nicht mehr a priori von einem Typ 1 ausgehen. Jeder zehnte juvenile Diabetiker in den USA hat einen Typ-2-Diabetes, viele zeigen bereits vor dem 20. Geburtstag Organschäden.}

— Immer mehr Kinder und Jugendliche erkranken an Diabetes: In den acht Jahren zwischen 2001 und 2009 wurde ein Anstieg der juvenilen Typ-1-Diabetiker um 23\% sowie ein Anstieg der juvenilen Typ-2-Diabetiker um 21\% registriert.

Nach Daten der "SEARCH for Diabetes in Youth Study "wird die Anzahl der Kinder und Jugendlichen mit Diabetes in den USA auf 189000 geschätzt, von denen ca. 20000 an einem Typ 2 erkrankt sind.

\section{Organschäden bei 18-jährigen}

Wie Frau Dr. Giuseppina Imperatore vom Center for Disease Control and Prevention in Atlanta berichtete, haben die Patienten mit so früher Diabetesdiagnose nicht nur ein hohes Risiko, im Laufe des noch langen Lebens Diabeteskomplikationen zu entwickeln. Bei vielen manifestieren sie sich bereits im Jugendalter. „Wir fanden bei unseren jun-

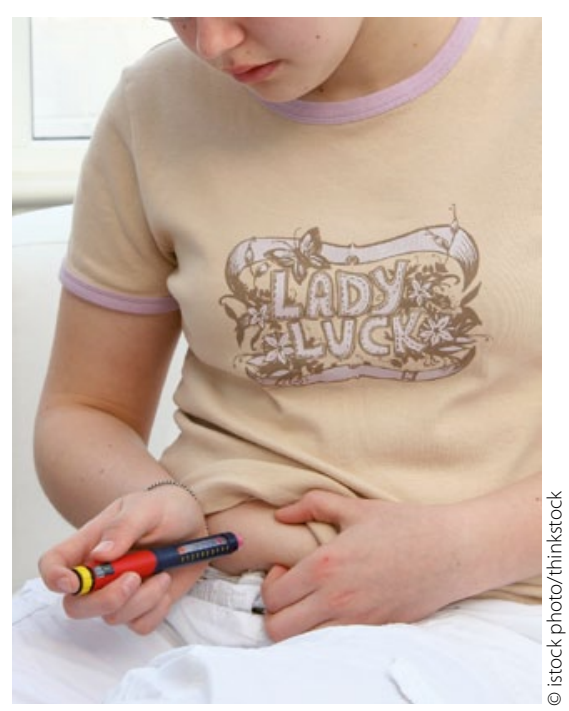

Jeder zehnte jugendliche Diabetiker ist ein Typ 2. gen Patienten bereits nachweisbare Schäden an den peripheren sowie an den autonomen kardiovaskulären Nerven“, berichtete die Epidemiologin. Manche Jugendliche, v. a. solche mit Typ-2-Diabetes, wiesen bereits eine Proteinurie als Zeichen einer diabetischen Nierenschädigung auf.

Bei Jugendlichen mit Typ-2-Diabetes schreitet die Erkrankung schneller fort, führt früher zu Komplikationen, und sie werden schneller insulinpflichtig. Dies folgt aus der "Treatment Options for Typ 2 Diabetes in Adolescents and Youth Study". In dieser Studie waren 699 jugendliche Typ-2-Diabetiker zwischen 10 und 17 Jahren in drei Gruppen behandelt und 2-6 Jahre nachverfolgt worden.

\section{Sehr rasche Insulinpflicht}

„Die gute Nachricht lautet, dass etwa 50\% der Kids früh und gut auf Metformin an-
Ist-Status bei Typ-1-Diabetikern
Vor allem Kinder und Jugendliche mit Typ-1-Diabetes sind oft sehr schlecht eingestellt, wie jetzt die US-RegisterStudie T1D-Exchange mit 25000 Typ1-Diabetikern, darunter 13000 Kinder und Jugendliche, zeigt. Die alarmierenden Trends im Einzelnen: gut: Kinder bis 13 haben im Schnitt $\mathrm{HbA}_{1 \mathrm{c}}$-Werte von 8,3\%, Jugendliche von $8,6 \%$ und Erwachsene über 26 Jahre von $7,6 \%$.

2 Die Stoffwechselqualität korrelierte eindeutig mit der Häufigkeit der täglichen Blutzucker-(BZ-)Selbstkontrollen. Patienten mit 0-2 Messungen täglich hatten einen $\mathrm{HbA}_{1 \mathrm{c}}$ von 9,3\%,
(1) Die Stoffwechselkontrolle ist nicht sprechen und mit dieser Therapie dann erst einmal stabil eingestellt sind“', berichtete Phil Zeitler, Pädiater an der University of Colorado. Die anderen 50\% der jungen Patienten müssen bereits nach wenigen Krankheitsjahren mit Metformin plus Insulin behandelt werden. Die vorliegende Studie untersuchte zwar Rosiglitazon als Therapiealternative, welche heute aber nicht mehr empfohlen werden kann.

Laut Zeitler ist es unbedingt notwendig, weitere Therapiealternativen für jugendliche Typ-2-Diabetiker zu erforschen. Denn viele der Patienten leiden bereits an diabetischen Komorbiditäten. „Fast ein Drittel der jungen Patienten wies nach nur vierjähriger Diabetesdauer eine Hypertonie auf, 17\% schieden Albumin mit dem Urin aus, $13 \%$ zeigten diabetestypische Veränderungen an der Retina. „In 10 bis 20 Jahren“, so Zeitler, „werden viele dieser Kinder ernsthafte gesundheitliche Probleme bekommen". Sie sind dann gerade über 30 Jahre alt. DR. MED. DIRK EINECKE -

- Quelle: 72. Scientific Sessions 2012, American Diabetes Association, Philadelphia, 8.-12. Juni 2012

\section{Schlecht eingestellt, zu dick, häufig unterzuckert}

solche mit 3-4 Messungen von 8,5\%. Wer täglich mindestens 7-mal den BZ bestimmte, lag mit dem $\mathrm{HbA}_{1 c}$ unter $8 \%$.

3 Der Typ-1-Diabetes ist nicht länger eine Erkrankung schlanker Patienten: Adipös oder übergewichtig sind $30 \%$ der Kinder, 40\% der Jugendlichen und 65\% der erwachsenen Typ-1-Diabetiker.

(4) Stoffwechselentgleisungen sind häufig: $4 \%$ (über 50-Jährige) bis 10\% (13- bis 26-J.) der Patienten erleiden jährlich eine Ketoazidose. Schwere Hypoglykämien, die zu Anfällen oder Koma führen, erleiden $8 \%$ aller Typ-1-Diabetiker mit einer Krankheitsdauer unter 20 Jahren. Mit längerer Krankheitsdauer steigt die jährliche Inzidenz schwerster Unterzuckerungen auf ca. $20 \%$ an. DE 